\title{
Análisis del Desarrollo Innovador para el Aprovechamiento de la (Guadua angustifolia Kunth) en la Sustitución de Cultivos Ilícitos
}

\section{Analysis of Innovative Development for the Use of (Guadua angustifolia Kunth) in the Substitution of Illicit Crops}

Fecha de Recepción: 12.02.2020

Fecha de Aceptación: 10.05.2020

Doi: https://doi.org/10.19053/01217488.v11.n2.2020.11518

\author{
Céspedes Prieto Nubia Edith ${ }^{\mathrm{a}}$ \\ Carda Castello Juan ${ }^{\text {b }}$ \\ Cervantes Estrada Luis Carlos ${ }^{c}$ \\ Gil Noreña Jonnathan Manuel ${ }^{\mathrm{d}}$
}

\section{Resumen}

Con la firma de los acuerdos de paz de 2016, uno de los problemas que se manifiesta, es el aumento considerable de cultivos ilícitos, especialmente en zonas fronterizas de Colombia como Cauca, Nariño y Norte de Santander. Teniendo en cuenta la estrategia de implementación de los acuerdos en materia de sustitución de cultivos ilícitos y reforma rural integral, en este trabajo se presenta una propuesta alternativa de implementación integral y sostenible del cultivo de (Guadua angustifolia, Kunth) y la oportunidad de innovación que tiene la guadua para la producción de materiales con valor agregado, ofreciendo una oportunidad de mejora en la calidad de vida para el Pacífico colombiano. Se hace una revisión documental relacionada con las realidades y desafíos que plantea la implementación de los acuerdos de paz, se establece que el cultivo y aprovechamiento de la guadua es una estrategia viable y sostenible para la erradicación voluntaria de cultivos de coca encamina al desarrollo territorial de Tumaco, Nariño aplicando una estrategia basada en cuatro ejes fundamentales: 1) Participación comunitaria 2) Estudio, socialización e implementación del cultivo de la guadua como estrategia de reemplazo 3) Desarrollo industrial de productos de guadua, 4) Acompañamiento de las fuerzas de seguridad y la Policía Nacional y 4) Monitoreo y asistencia permanente del Estado.

\begin{abstract}
With the signing of the 2016 peace agreements, one of the problems that manifests itself is the considerable increase in illicit crops, especially in Colombian border areas such as Cauca, Nariño and Norte de Santander. Taking into account the implementation strategy of the agreements regarding the substitution of illicit crops and comprehensive rural reform, this work presents an alternative proposal for the comprehensive
\end{abstract}

a Dra. Ciencias Química, Universidad Jaume I. España; Magister Ciencias Química, Universidad Nacional de Colombia, Especialista en Gestión Ambiental Universidad Distrital, Ingeniera Ambiental, Lic. Química y Biología, Investigador Asociada, Escuela de Cadetes General Santander - Policía Nacional de Colombia.

* $\quad$ Autor de Correspondencia necespedesp@unal.edu.co

b Dr. en Ciencias Químicas, Lic. en Ciencias Químicas, Profesor Titular de la Universitat Jaume I, España, Director de la Cátedra de Innovación Cerámica "Ciutat de Vila-real” - España. autor/coautor de varias patentes, algunas con explotación industrial.

c Magister en Docencia Universitaria, Universidad Sergio Arboleda, Administrador Policial, Jefe de Investigación Escuela de Cadetes General Francisco de Paula Santander.

d Biólogo-Ornitólogo, Universidad del Tolima, Administrador Policial de la Escuela de Cadetes General Francisco de Paula Santander, Subteniente de la Policía Nacional de Colombia 


\author{
Nubia Edith Céspedes Prieto - Juan Carda Castello \\ Luis Carlos Cervantes Estrada - Jonnathan Manuel Gil Noreña
}

\begin{abstract}
and sustainable implementation of the cultivation of (Guadua angustifolia, Kunth) and the opportunity for innovation. that guadua has for the production of materials with added value, offering an opportunity to improve the quality of life for the Colombian Pacific. A documentary review is made related to the realities and challenges posed by the implementation of the peace agreements, it is established that the cultivation and use of guadua is a viable and sustainable strategy for the voluntary eradication of coca crops aimed at the territorial development of Tumaco, Nariño applying a strategy based on four fundamental axes: 1) Community participation 2) Study, socialization and implementation of the cultivation of guadua as a replacement strategy 3) Industrial development of guadua products, 4) Accompaniment of the security forces and the National Police and 4) Monitoring and permanent assistance from the State.
\end{abstract}

Palabras clave: reforma rural integral, Guadua angustifolia Policía Nacional, sostenibilidad.

Keywords: Comprehensive rural reform, Guadua angustifolia, National Police, Sustainability.

\section{INTRODUCCIÓN}

Dos de los puntos cardinales abordados en el acuerdo final que tiene como propósito la terminación del conflicto y la construcción de una paz estable y duradera en Colombia, son el de la Reforma Rural Integral y la Sustitución de Cultivos Ilícitos [1]. En estos puntos del acuerdo, se aborda con especial énfasis el rol activo de las comunidades y la ciudadanía, para la formulación de políticas públicas y los planes de desarrollo a nivel local y nacional en la restitución de tierras a las víctimas del conflicto armado y la erradicación manual y voluntaria de los cultivos ilícitos [2].

Así pues, la solución definitiva a la cuestión de las drogas ilícitas y el narcotráfico que plantea el acuerdo suscrito en La Habana, tiene como eje la erradicación de cultivos de coca en Colombia, los cuales, paradójicamente han experimentado una considerable expansión en los últimos años. El territorio afectado por coca en 2018 llegó a $141.125 \mathrm{~km} 2$, esto es el $63 \%$ del territorio afectado por coca en los últimos diez años y el $51 \%$ si se considera toda la serie histórica. Entre 2017 y 2018 se reporta una reducción del $11 \%$ en la coca; pero esto se enmarca en las dinámicas de erradicación manual sin embargo, la sostenibilidad de esta reducción Dicho incremento se presentó en las zonas que ya tenían la mayor concentración de cultivos desde 2015 y se expresó en un aumento del $52 \%$, del cual Tumaco (Nariño) resultó ser la región más afectada, con un incremento del 18\%[3]. Por ende, la región del Pacífico se presenta como una de las zonas del país con mayor incidencia de este tipo de cultivos, debido a la militancia a lo largo de su territorio de grupos guerrilleros cuya principal fuente de financiación es la exportación de droga (cocaína). La cual, de una u otra manera representa algún lucro para quienes se dedican a su siembra y procesamiento. Por esta razón, cultivos alternativos y nuevos métodos del manejo de tierras ofrecen una oportunidad para el restablecimiento del orden público y mejores condiciones para el desarrollo económico y social de la región [3].

Dentro del desarrollo de planes diseñados por el gobierno para la solución del problema de lao cultivos ilícitos en el posconflicto, aparte de unos recursos estimados en más de 120 billones de pesos [4] se plantean tres métodos de restitución de tierras e iniciativas, que incluyen en primer lugar el desarrollo y la generación de conciencia de la población respecto a las problemáticas que este ejercicio trae consigo y en segundo y tercer lugar, está el desarrollo y la implementación de proyectos agrícolas en las zonas más afectadas, con la inclusión de la comunidad en cada uno de los niveles de desarrollo de dicho plan [3].

En esta perspectiva, aquí se propone la implementación integral y sostenible del cultivo de guadua (Guadua angustifolia, Kunth) como herramienta de innovación en la producción de materiales para mejorar la calidad de vida de las comunidades más afectadas por el uso de cultivos ilícitos en la región de Tumaco, Nariño, en el Pacífico colombiano. Además, se propone con esto un eje de defensa y seguridad de la Policía Nacional, pues con la implementación exitosa de esta economía de transición en el posconflicto, se abonará terreno en la garantía a una paz estable y duradera en una región asolada por la guerra en el país, que tiene distintos actores armados, entre los que se destacan la guerrilla y el paramilitarismo, que detentan el poder sobre los cultivos ilícitos y el narcotráfico [5].

Guadua angustifolia es una especie de origen centroamericano con gran importancia desde el 
punto de vista económico y social [6] Estudios demuestran que las especies de bambú son conocidas como el 'acero vegetal', ya que, al estar expuestas a diversas pruebas estructurales, dan cuenta de una alta resistencia a la tracción y compresión[7] Guadua es un tipo de bambú que se ha convertido en una alternativa para mitigar los efectos causados por el cambio climático [6] Con esto, la sostenibilidad ambiental es un indicador positivo y constituye un avance importante en la definición de sostenibilidad de los bosques de guadua. Esta condición de sostenibilidad también implica mantener el manejo de la intensidad actual y las condiciones específicas de estos bosques, los cuales, con dinámicas importantes, responden adecuadamente a los niveles de cosecha. Este material vegetal se usa en la construcción de viviendas, cobertizos, galpones, almacenes, casas de campo, etcétera. Sus propiedades mecánicas son importantes para todo material compuesto, porque permiten definir un uso final del producto de acuerdo con su resistencia a la tensión, flexión e impacto[8].

Cabe destacar que Colombia es un país pionero en el uso estructural del bambú y en el desarrollo de tecnologías constructivas[7] y hasta el momento es el único país de América que ha elaborado una serie de normas de calidad para su cultivo. Además de la sustitución de cultivos ilícitos en zonas de afluencia como Tumaco, que es considerada actualmente como la capital de la coca en la región Pacífica de Colombia[3] Por ello, con este proyecto también se busca eliminar esa percepción cocalera de la región, que, con su gente trabajadora y con ganas de cambiar su situación económica, mediante el auspicio del Gobierno Nacional y de sus cuerpos de seguridad policiales, tracen una hoja de ruta para establecer mecanismos de compensación de las víctimas del conflicto, teniendo en cuenta la participación, evaluación, y sustitución de programas de recuperación ambiental, que tengan una perspectiva de supervivencia económica para dichas comunidades, de cara a una paz duradera después de la firma del acuerdo.

\section{METODOLOGÍA}

Este trabajo fue concebido como una investigación de tipo documental, que por medio de una revisión en bases de datos indexadas y a través de una búsqueda selectiva de artículos relacionada con la producción, desarrollo y aplicaciones de innovación teniendo como marco tres ejes: Con- flicto Colombiano, Sustitución de Cultivos ilícitos y desarrollo económico a partir de la guadua se hizo una revisión técnica y crítica. que orientará el conocimiento de la guadua como una alternativa viable y sostenible a ser implementada en la región de Tumaco, Nariño, por ser el departamento que mantiene los niveles más altos de producción de cultivos ilícitos; y que requiere de una estrategia de desarrollo territorial de mayor impacto, , y de cara a mitigar, en el largo plazo el impacto que los cultivos ilícitos han causado en la región ubicando, el rol de la Policía Nacional (dentro de las facultades legales del Estado) como organismo garante para mantener las condiciones de seguridad que se requieren y apoyar a través de la Dirección de Carabineros y el programa del Sistema de Servicio Técnico Rural a estas comunidades.

\section{Acuerdo de Paz y sustitución de cultivos ilícitos}

Según el acuerdo de producción y comercialización de drogas ilícitas que fue discutido en la Mesa de Negociaciones en la Habana, "para contribuir al propósito de sentar las bases para la construcción de una paz estable y duradera es indispensable encontrar una solución definitiva al problema de las drogas ilícitas". En tal sentido, la necesidad de una Reforma Rural Integral que resalte el enfoque de la denominada Agricultura Familiar deberá estar articulada con los programas de desarrollo alternativo, en aquellas zonas históricamente afectadas por el conflicto armado y el narcotráfico. En 2017, el gobierno de Colombia puso en marcha el Programa Nacional Integral de Sustitución de Cultivos de Uso Ilícito (PNIS) como un componente de la Reforma Rural Integral. El programa se concibió para apoyar la transición de los cultivadores a la legalidad con un esquema sustentado en subsidios familiares asignados por el gobierno, la implementación de un Plan de Acción Inmediata (PAI) y la ejecución de los Planes Integrales Comunitarios y Municipales de Sustitución y Desarrollo Alternativo (PISDA)[9], [10] La idea central del programa es la sustitución voluntaria de cultivos de uso ilícito así como promover las condiciones que permitan a las comunidades involucradas disfrutar de una calidad de vida, proporcionando a las personas vinculadas con el cultivo de ilícitos? ya sea como mano de obra o propietarios de parcelas o fincas oportunidades para abandonar esa actividad y evitar la reincidencia [11]. 


\section{Nubia Edith Céspedes Prieto - Juan Carda Castello \\ Luis Carlos Cervantes Estrada - Jonnathan Manuel Gil Noreña}

Siguiendo la información de la Alta Consejería Presidencial para el Posconflicto, Derechos Humanos y Seguridad, luego de la firma de estos acuerdos colectivos (Fase A) y de su socialización (Fase B), el PNIS se pone en marcha (Fase C) con la inscripción de las familias, el inicio del proceso de sustitución y la entrega de beneficios económicos (36 millones COP anuales/familia restituida) para los cultivadores que cumplan con lo pactado. Esta fase contempla de otra parte la caracterización familiar, la realización de asambleas comunitarias y la conformación de la estructura territorial del Programa[11].

Según los datos de la Agencia Nacional de Tierras (ANT), hasta la fecha hay registradas en nombre de la Nación 200.000 hectáreas de los 3 millones que se espera llevar al Fondo de Tierras hasta 2028. De este fondo saldrán las hectáreas de los campesinos que no poseen tierra o han sido desposeídos por la violencia. Según el balance entregado en 2018, han sido formalizadas 1'470.000 hectáreas de la meta de 7 millones proyectados para los próximos 10 años. De estas tierras formalizadas a la fecha se han entregado 3220 títulos de propiedad y la adjudicación de 22.843 baldíos[12].

A estos puntos también hay que añadir la inclusión de vías terciarias y de transporte que compondrían la cadena de producción. Según datos del Gobierno Nacional, este ha intervenido $1780 \mathrm{Km}$ de vías terciarias de los cerca de 2400 que espera intervenir para que los campesinos puedan comercializar sus productos, sin depender de intermediarios [12].

Teniendo en cuenta el desarrollo sostenible por el que deberá propender la estrategia de erradicación de cultivos ilícitos, a este respecto debe añadirse la delimitación de la frontera agrícola con el objeto de proteger las áreas de interés ambiental, así como el uso y la distribución de los suelos a lo largo del territorio nacional Razón por la cual, el gobierno de Colombia se comprometió en un plazo no mayor de dos años a trazar un plan de zonificación ambiental "que delimite la frontera agrícola y entregue la cartografía de: zonas de reserva forestal, zonas de alta biodiversidad, ecosistemas frágiles y estratégicos, cuencas, páramos, humedales, y demás fuentes de recursos hídricos con miras a proteger la biodiversidad...[12]".

\section{Panorama de los Cultivos de Coca}

Colombia se plantea varios desafíos, nacionales e internacionales, en la erradicación exitosa y duradera de los cultivos ilícitos y en especial, de los cultivos de coca. A pesar de los acuerdos firmados, la cifra de cultivos en el país sufrió un incremento excepcional al pasar de 96.000 hectáreas en 201546.000 hectáreas en 2016y para el 2018, estos reportan una disminución en el 11\%. Este crecimiento de cultivos de coca se presentó en las zonas que ya tenían la mayor concentración de cultivos desde 2015 y tuvo un incremento neto del $52 \%$ La mitad de ese incremento está concentrado en dos departamentos fronterizos: Norte de Santander y Nariño. Y junto con Putumayo, se concentra más del $60 \%$ de la coca que se produce en el país. Tumaco es el municipio más afectado por cultivos de coca en todo el territorio nacional y ha permanecido en la lista de los más afectados desde 2002[3].

Vale la pena mencionar que en las regiones de Norte de Santander, Cauca-Nariño y la región Central han operado diferentes actores ilegales, entre los que se destacan disidencias de las FARC, EPL, BACRIM y ELN. Así pues, la dinámica y el incentivo del precio de la hoja de coca se ha mantenido por parte de estos grupos desde 2015 y ha sido un escollo principal tras la firma de los acuerdos, de cara al posconflicto. En 2016 el precio de la hoja de coca se mantuvo alto, pasando de 2.014 COP en 2013 a 2.900 COP en 2016 [3].

En el trascurso de los últimos años y previo a la firma de los acuerdos se han implementado diversos programas de sustitución de cultivos ilícitos en Nariño y en particular en algunas zonas veredales de Tumaco. La experiencia mostró que Nariño tuvo especial acogida por los cultivos de café (59\%), en contraste con otros departamentos con alta concentración de cultivos de coca, como Norte de Santander que enfatizó en la ganadería poco sostenible, o Guaviare que en la medida de sus condiciones optó por los sistemas agro-silvopastoriles además de cultivos permanentes, aunque en menor proporción de Cacao, Plátano y Caucho. Sin embargo, se presentaron algunos problemas relacionados con la cadena de distribución y consumo, y la falta de garantías estatales que no regulaban el precio de las materias primas y el agro insumo (Santamaría, 2015). Basados en esta experiencia, se prevé que una vez puesto en marcha el Programa Nacional 
Nubia Edith Céspedes Prieto - Juan Carda Castello

Luis Carlos Cervantes Estrada - Jonnathan Manuel Gil Noreña

Integral de Sustitución de Cultivos de Uso Ilícito (PNIS) estas falencias se superen.

En el marco de la implementación de alternativas viables y sostenibles también es necesario ubicar el rol de las Zonas de Reserva Campesinas (ZRC) en el posconflicto; ya que se trata de iniciativas agrarias que tienen como objetivo contribuir a la paz en un marco de desarrollo sostenible. Para esto, el gobierno e instituciones como la policía comunitaria promoverán la participación activa de las comunidades que habitan las ZRC en la ejecución de sus planes de desarrollo, teniendo en cuenta una delimitación estricta en la posesión de tierras y de títulos que no entren en conflicto con zonas estratégicas como parques nacionales o reservas forestales [9].

\section{Estrategias novedosas para la guadua}

Los bambúes pertenecen a la Familia Poaceae, una de las familias de plantas más extensas y con mayor éxito evolutivo. La familia comprende cerca de 700 géneros y aproximadamente 10000 especies en la que destaca la subfamilia Bambusoideae, con más de 80 géneros y 1000 especies registradas[13].

Los bambúes son consideradas plantas evolucionadas, por sus adaptaciones y pueden ser leñosos o herbáceos, de hábito erecto o escandente y con tamaños que oscilan desde los 5 metros hasta los 30 metros de altura. Latitudinalmente, los bambúes se distribuyen desde los $46^{\circ}$ Norte hasta los $47^{\circ}$ Sur, y altitudinalmente se extienden desde el nivel del mar hasta $4000 \mathrm{~m}$. Prefieren ambientes húmedos como los de la selva tropical y subtropical, pero se adaptan también en regiones secas[14].

Alrededor del 85\% de los bambúes herbáceos del mundo se encuentran en el Neotrópico. Se distribuyen desde México hasta Argentina, siendo Brasil el país más rico en géneros y especies. Estos bambúes tienen culmos herbáceos, sistema simple de ramificación, sistema rizomático simple, floraciones frecuentes, no cíclicas, y crecen generalmente en el sotobosque de la selva tropical y subtropical por debajo de los $1500 \mathrm{~m}$, donde son polinizados principalmente por especies de insectos[15].

En Colombia existen 11 de los 22 géneros (50\%) de bambúes herbáceos que hay reportados hasta el momento para el nuevo mundo y aproximadamente
25-30 de las 130-140 especies de este continente, estas pertenecen a los géneros Cryptochloa, Lithachne, Maclurolyra, Olyra, Pariana, Parodiolyra, Piresia, y Raddiella, de la tribu Olyreae, Pharus de la tribu Phareae, Streptochaeta de la tribu Streptochaetea y Streptogyna de la tribu Streptogyneae. Asimismo, se han reportado 45 especies reunidas en 7 géneros: Arthrostylidium, Aulonemia, Chusquea, Elytrostachys, Guadua, Neurolepis y Rhipidocladum; creciendo en diversos tipos de hábitats: hay géneros como Guadua en la zona de vida bosque muy húmedo tropical (bmh-T), como Rhipidocladum y Elytrostachys en la zona de vida bosque seco tropical (bs-T), y en lugares fríos y húmedos del páramo como Chusquea $\mathrm{y}$ Neurolepis[15].

El género Guadua es nativo del Neotrópico. Se extiende desde México por todos los países de Centro América y Sudamérica hasta Argentina, con excepci6n de Chile. Incluye unas 28 especies de las cuales cuatro especies y una variedad son significativas para Colombia: G. amplexifolia, G. angustifolia, G. angustifolia var. bicolor, G. superba, y G. weberbaueri [14]

El género Guadua alcanza su mayor grado de desarrollo bajo condiciones mesofíticas en alturas que no exceden los $1500 \mathrm{~m}$ (McClure, 1973). La temperatura es factor limitante en su distribución latitudinal y altitudinal; no resiste periodos prolongados por debajo de $0^{\circ} \mathrm{C}$ y no crece bien en áreas donde la precipitación es menor de $1200 \mathrm{~mm}$ anuales. Temperaturas por debajo de $11^{\circ} \mathrm{C}$, con duraciones mayores de 6 horas diarias, son un limitante para: su desarrollo (Londoño, X.; Prieto, L., 1983). En Colombia, la mayoría de las poblaciones de Guadua cree en entre 0-1000 m, ocupando diversos hábitats en las formaciones vegetales bosque húmedo tropical (bh-T) y bosque muy húmedo pre montano (bmhPM). G. amplexifolia domina en la planicie norte de la Costa Atlántica, en la zona de vida bosque seco tropical (bs-T), en donde forma pequeñitas manchas, a orillas de ríos 0 quebradas, entre $0-500$ $\mathrm{m}$ de altitud. Guadua angustifolia presenta el más amplio rango de distribución, se extiende por las 3 cordilleras, desde el norte hasta el sur, a alturas entre 500-1500 m (c.1800 m), predominando en los valles interandinos, donde forma grandes sociedades llamadas "guaduales"; la Guadua angustifolia var. bicolor se localiza en la zona central de los Andes, y se ha observado únicamente en la vertiente oriental de la cordillera Occidental y 
Nubia Edith Céspedes Prieto - Juan Carda Castello

Luis Carlos Cervantes Estrada - Jonnathan Manuel Gil Noreña

en la vertiente occidental de la cordilleras Central y Oriental. Las especies G. superba y G. weberbaueri son netamente amazónicas, crecen al suroriente de la Amazonia, por debajo de los 500m [6].

\section{La guadua como producto sostenible}

Los sistemas constituidos por G. angustifolia se caracterizan por tres componentes principales: 1) En la vegetación asociada predominan especies de la familia Piperaceae, Rubiaceae, Araceae, Mimosaceae, Compositae, y algunos pteridófitos. 2) Las especies asociadas en su mayoría están ubicadas en el sotobosque y 3) Los cambios en las condiciones del desarrollo del guadual ocasionan cambios en su vegetación. Zonas afectadas por el fuego o manejo intensivo se ven invadidas por plantas heliófilas como las Poaceae, Asteraceae y Convolvulaceae. Así pues, La vegetación asociada es un elemento clave que ayuda a conservar la humedad del suelo y del ambiente hacia el interior del guadual, aportando materia orgánica, alimento y refugio a la fauna que pudiera estar presente[6].

La guadua aporta a los contextos ambientales una oportunidad de atención a situaciones puntuales como la disminución en la huella de carbono, debido al tiempo tan corto de regeneración y facilidad de producción, y a nivel de las construcciones el confort climático y acústico que las viviendas de guadua ofrecen. [16]

Los rizomas y hojas en descomposición conforman en el suelo un sistema de absorción, evitando que el agua fluya de manera continua, con lo cual se propicia la regulación de los caudales y la protección de la erosión del suelo. El sistema entretejido de rizomas y raíces origina una ma1la, que les permite comportarse como eficientes muros biológicos de contención que controlan la socavación lateral y amarran fuertemente el suelo, previniendo la erosión y haciendo de la guadua una especie con función protectora, especial para ser usada en sistemas de galería de cuencas hidrográficas[16].

\section{Impacto económico y social de la guadua en Colombia}

La "guadua colombiana" o G. angustifolia es la especie forestal con mayor proyección económica, puesto que, empleada como materia prima, su utilización en la construcción y la industria permite reducir costos debido a sus excelentes propiedades físicas y químicas. De ahí que el bambú-guadua sea conocido como el oro verde del siglo XXI y se haya convertido en una alternativa para suplir los efectos del cambio climático y como una solución energética para el gran déficit actual (Londoño, X., 2011). En Colombia la guadua ha acompañado el desarrollo de culturas prehispánicas hasta la actualidad.

Guadua angustifolia es ampliamente utilizada en aplicaciones estructurales debido a su alta resistencia y atributos dendrométricos (por ejemplo longitud y diámetro del culmo). De hecho, las casas de guadua, los puentes y demás estructuras en casas rurales son ampliamente conocidos a nivel mundial[17] [18].

Aunque se encuentra en casi todo el país, la guadua ha estado culturalmente ligada a la zona andina, especialmente en el Eje Cafetero que comprende cinco departamentos en las montañas y valles del centro-occidente del país. Hoy día existe un interés mundial por el desarrollo y cultivo del bambú gracias a la coyuntura mundial de la escasez existente en el mercado de madera y energía. Gracias a la calidad de su fibra y a su tamaño, la guadua ofrece una serie de ventajas con respecto a la madera que la hacen más competitiva e interesante como lo son, a saber: a) sostenibilidad en el tiempo, b) auto-propagación c) altas ratas de crecimiento (11-21 cm/ día), d) alta productividad, e) generación intensa de mano de obra en toda la cadena de producción [13].

El mercado nacional y mundial del bambú ha mostrado una creciente demanda desde 2014. La guadua, como ocurren en otros países con otros bambúes, bien podría configurarse en una parte importante y sostenible dentro de la economía colombiana. Según datos del INBAR, el mercado mundial de diversos productos de bambú movió cerca de 3.200 millones de dólares en 2011; en donde China aparece como el principal productor, debido a fuertes políticas públicas nacionales de incentivos al cultivo, cosecha y generación de valor agregado [19]. A partir del precio comercial pagado en depósito de madera (guadua sin valor agregado), la comercialización del culmo alcanzó 5.7 millones de dólares en 2011. No obstante esta cifra representa apenas un $1 \%$ del valor estimado del comercio anual de madera en Colombia (Cardona, 2014). A pesar del enorme 
Nubia Edith Céspedes Prieto - Juan Carda Castello

Luis Carlos Cervantes Estrada - Jonnathan Manuel Gil Noreña

potencial, en Colombia se estiman apenas unas 56.000ha de guadua, comparadas con las demás plantaciones forestales con fines comerciales, que no siempre garantizan sostenibilidad y alcanzan las 200.000 ha [5].

A pesar de estas dificultades se han encarado numerosos esfuerzos por consolidar la producción, el comercio y los derivados de la guadua en el país, mediante diversas estrategias que incluyen una mejor regulación estatal, presencia de capitales privados y flexibilización en el otorgamiento de licencias ambientales. Así como el desarrollo y la implementación de una política pública para la guadua, incluida la creación del Consejo Nacional del Bambú guadua [14].

\section{La cadena de producción y comercialización de Guadua angustifolia y sus derivados}

La comercialización de la guadua empieza a partir de la producción de propágulos (o chusquines) que en muchas regiones como Quindío y Risaralda ha alcanzado estándares internacionales, ante la demanda de la especie para aprovechamiento forestal. De otro lado, comprende la comercialización de la guadua, luego de la transformación primaria de diversos productos de los cuales dependen procesos semi-industriales e industriales, que pueden ser para la construcción de viviendas, muebles o artesanías.

La producción de propágulos de guadua se realiza en el banco de propagación o en el vivero forestal y comprende tres fases:

Establecimiento del banco de propagación. Se selecciona un área con suelos francos y buena humedad, preferiblemente con presencia de cuencas en sus alrededores. Se procede con el trazado, que es disponer del material según un sistema de siembra y una distancia entre propágulos que determinan la densidad poblacional de los mismos en el área de siembra. Posteriormente, se ahoya cada punto trazado de una dimensión según el método de propagación seleccionado.

El método más usado y exitoso es el de reproducción asexual, que puede realizarse por medio del rizoma sin culmo; transporte directo, a través del rizoma y parte del tallo; por segmentos del tallo; ramas no lignificadas; acodos o por riendas laterales. La segunda fase consiste en el manejo del banco de propagación (plateo de $30 \mathrm{~cm}$ de diámetro), riego y abonamiento. La tercera fase consiste en el trasplante de los chusquines a la bolsa con tierra fertilizada que posteriormente serán llevados a la zona de siembre definitiva. Posterior a eso se traza el Plan de Establecimiento y Manejo Forestal que comprende las fases de establecimiento de la plantación y el manejo de guaduales naturales y plantados, para su posterior aprovechamiento que consiste en la elección de cortes dependiendo de la industria.

\section{Aspectos organizacionales, ocupacionales y de innovación en el uso de la guadua}

Aunque en la actualidad hay ausencia de censos sobre la cadena productiva y la cantidad de personal empleado, se han hecho estudios prospectivos sobre la generación de empleo que pude derivarse del uso del "acero vegetal". La esterilla y la guadua rolliza son productos de alta demanda en el mercado nacional, pues hoy por hoy existen depósitos de madera que son vendidos de manera permanente, para la construcción y otros usos.

Así pues, se necesita un fortalecimiento de la cadena productiva de la guadua, con el objeto de lograr un reconocimiento del sector como actividad económica en el marco de generación de empleo en el país, y porque no, proponer ideas innovadoras que permitan hacer uso de hasta la última propiedad disponible del material empleado para las ya mencionadas aplicaciones; lo anterior gracias a las propiedades fisicoquímicas de este material vegetal (Tabla 1). Ciertas actividades productivas, económicas y comerciales son desarrolladas con base en la obtención del material y el procesamiento del mismo, donde se observan desde la propagación en viveros, el establecimiento de plantaciones, artesanías, confección de muebles y textiles, construcción entre otros, procesos que a su vez, tienen un impacto positivo en la demanda de personal y la producción de empleo que satisface las necesidades de un ciclo productivo sostenible como lo es el cultivo y aprovechamiento de la Guadua angustifolia Kunth como un producto con valor agregado para la superación de las brechas generadas por el postconflicto y la sustitución de los cultivos ilícitos en el país (Tabla 2). [15][6] 
Nubia Edith Céspedes Prieto - Juan Carda Castello

Luis Carlos Cervantes Estrada - Jonnathan Manuel Gil Noreña

Tabla 1. Importancia de Guadua angustifolia Kunth

\begin{tabular}{|c|c|}
\hline $\begin{array}{l}\text { Propiedades físicas } \\
\text { (Unidades en } \mathrm{Kg} / \mathrm{cm}^{2} \text { ) }\end{array}$ & $\begin{array}{l}\text { Módulo de elasticidad a tracción: } 190000 \text {; Otras maderas: } 90000 \text { y } 180000 \\
\text { Módulo de elasticidad a compresión: 184000; Otras maderas: } 96000 \text { y } 169000 \\
\text { Módulo de elasticidad a flexión: } 179000 \text {; Otras maderas: } 108000 \text { y } 128000\end{array}$ \\
\hline Importancia ambiental & $\begin{array}{l}\text { Control de erosión, restauración de zonas degradadas, regulación de caudal hídrico (amortigua- } \\
\text { ción), aporte de materia orgánica, refugio de fauna y flora, fijación de carbono, ecoturismo, pai- } \\
\text { sajismo, protección de suelos debido a su sistema de raíces, regulación de la humedad del bosque, } \\
\text { clave para proporcionar refugio a especies vulnerables como anfibios y otros microhábitats. Ade- } \\
\text { más, de aplicaciones para la producción de agentes de protección ambiental, pesticidas y agentes } \\
\text { químicos para el control de flora asociada a plantaciones de uso comercial }\end{array}$ \\
\hline $\begin{array}{c}\text { Importancia económica } \\
\text { y sociocultural }\end{array}$ & $\begin{array}{l}\text { Mercado que representa unos } 17 \text { mil millones de dólares anuales } \\
\text { Altos rendimientos en volúmenes por hectárea, rápidas tasas de crecimiento y aprovechamiento } \\
\text { por lo que es aprovechada como materia prima, importante fuente de pulpa para la industria del } \\
\text { papel, garantizando sostenibilidad para otras especies maderables y vulnerables, Innumerables } \\
\text { estructuras para el campo de la construcción dadas sus condiciones de altura, durabilidad y resis- } \\
\text { tencia, además de ser un material que facilita su uso arquitectónico. En el sector de las artesanías } \\
\text { la elaboración de muebles, tamizados y láminas. En el sector agropecuario es usado en la cons- } \\
\text { trucción de cercas, corrales, cultivos, acueductos, etc. }\end{array}$ \\
\hline $\begin{array}{l}\text { Derivados potenciales } \\
\text { por compuesto químico } \\
\text { (Industria química) }\end{array}$ & $\begin{array}{l}\text { Tetraetil silicato: Revestimiento, anticorrosivo secador, antioxidante antirradical. Aplicados en } \\
\text { las industrias de construcción, arqueología y cosmética. } \\
\text { Diacetona alcohol: Solvente químico, Conservante, anticongelante, barniz (capa aislante), com- } \\
\text { ponente intermedio. Aplicados en las industrias eléctrica, química, farmacéutica y automotriz. } \\
\text { Dietilenglicol: Anticongelante, espesante sustitutivo, humectante, plastificante y solvente, aditi- } \\
\text { vo auxiliar, solvente secundario, resina de poliéster. Aplicados en las industrias textilera, farma- } \\
\text { céuticas, cosmética, papelera, cementera y automotriz. } \\
\text { Flavonoides: Antioxidantes, proliferación de células. Aplicados en la industria cosmética. } \\
\text { Entre otros compuestos químicos (metabolitos secundarios) con importancia antibiótica, antia- } \\
\text { lérgica y anticancerígena. }\end{array}$ \\
\hline
\end{tabular}

Fuente:

Tabla 2. Demanda de personal de la guadua por actividad económica. [6]

\begin{tabular}{|c|c|}
\hline Actividad & Demanda de personal \\
\hline $\begin{array}{c}\text { Propagación y viveros } \\
\text { Establecimiento de } \\
\text { plantaciones }\end{array}$ & $\begin{array}{r}\text { Profesionales técnicos y tecnólogos capacitados para realizar control de calidad en el material } \\
\text { forestal que se obtiene en los viveros, ej., propagación in vitro. }\end{array}$ \\
\hline $\begin{array}{c}\text { Aprovechamiento } \\
\text { Presenal técnico preparado para supervisar las obras que realizan las cuadrillas } \\
\text { encargadas de las obras de trazado, siembra y ahoyado. }\end{array}$ & $\begin{array}{r}\text { Trabajador de mano de obra calificada en la intervención y manejo de guaduales, que realicen } \\
\text { inventarios forestales y conozcan de silvicultura de guadua. }\end{array}$ \\
\hline Artesanía & $\begin{array}{r}\text { Asesores en mercadeo y comercialización. Ingenieros industriales capacitados en construcción } \\
\text { de maquinaria especializada y como asesores en el manejo de esta. }\end{array}$ \\
\hline Muebles & $\begin{array}{r}\text { Asesores en mercadeo y comercialización. Ingenieros industriales capacitados en construcción } \\
\text { de maquinaria especializada y como asesores en el manejo de esta. }\end{array}$ \\
\hline Construcción & $\begin{array}{c}\text { Ingenieros civiles, arquitectos, tecnólogos en obras capacitados en construcción de guadua. } \\
\text { Mano de obra calificada. }\end{array}$ \\
\hline Laminados & $\begin{array}{c}\text { Asesores en mercadeo y comercialización. Ingenieros industriales capacitados en construcción } \\
\text { de maquinaria especializada y como asesores en el manejo de esta. Trabajadores calificados } \\
\text { para operarios de plantas de laminados. }\end{array}$ \\
\hline Asistencia & $\begin{array}{r}\text { Biólogos, Ingenieros y tecnólogos forestales capacitados en el manejo, aprovechamiento y } \\
\text { reposición de bosques de guadua. }\end{array}$ \\
\hline
\end{tabular}


Nubia Edith Céspedes Prieto - Juan Carda Castello

Luis Carlos Cervantes Estrada - Jonnathan Manuel Gil Noreña

\section{Industria prospectiva: derivados industriales de la guadua (biomateriales, etc.)}

Si bien Colombia está lejos de implementar una industria altamente tecnificada en sus cultivos tradicionales, es importante resaltar que, tras la demanda internacional de guadua en los últimos años, se han venido adelantando estudios que aumentan el valor agregado de este recurso en la industria de biomateriales que derivan en el empleo de fibras, laminados, productos artesanales y diversas formas estructurales. Por lo tanto, el potencial económico es enorme para Colombia y sus regiones en las que se desea reemplazar el uso de cultivos ilícitos, pues como se describió anteriormente, el país posee una variedad excepcional de especies de bambúes leñosos y herbáceos, y la división del trabajo, así como la generación de empleos se configuraría en un panorama prometedor.[20]

Entre las innumerables promesas de esta planta, debido a su alta pirolisis $G$. angustifolia se configura como un candidato excepcional en la producción de bioetanol y otros biocombustibles [21],[22] [23].

Las fibras naturales son las más promisorias para sustituir a las fibras sintéticas y otros polímeros, debido a que no son tóxicas, son no abrasivas, poseen baja densidad, mínimo impacto ambiental y son biodegradables, e incluso poseen propiedades mecánicas similares comparadas con otras fibras sintéticas, como las fibras de vidrio, carbono y Kevlar (Rajesh, Pitchaimani, \& Rajini, 2016). A pesar de los avances, las fibras naturales poseen ciertas limitaciones empleadas como agentes de reforzamiento en termoplásticos debido a su incompatibilidad fisicoquímica entre las fibras hidrofílicas y la matriz hidrofóbica, cuando son usadas como refuerzo en matrices poliméricas, como en el caso de la guadua [24].

Además, según el observatorio de Agro cadenas de Colombia y en concordancia con el Ministerio de Agricultura y Desarrollo Rural (MADR) respecto a estudios realizados en los últimos 25 años (periodo de mayor desarrollo de la industria en países orientales como 'China), dicha implementación de proyectos de producción sostenible aporta un valor agregado al material vegetal recolectado, generando buenos ingresos lo que mejora considerablemente la calidad y estilo de vida de la población colombiana. Dentro de las propuestas abiertas al mercado, que involucran la implementación de proyectos de innovación y aprovechamiento del material vegetal se pueden obtener a partir de estas plantas, metabolitos entre otras sustancias, que tienen su principal aplicación en la industria farmacéutica, cosmética y alimentaria [15].

Otros productos con valor agregado que derivan de la transformación de plantas de G. angustifolia y en concordancia con investigaciones realizadas [6] son: Vinagre de bambú Es un producto obtenido durante las etapas preliminares del proceso de producción del carbón a partir de tallos y residuos de la planta. En este proceso el vapor producido de se condensa y recolecta como vinagre de bambú [25]. Dentro de los principales usos cosméticos de este producto se encuentra el tratamiento de la dermatitis atópica entre otras enfermedades de la piel; además de sus propiedades antiinflamatorias, antibióticas (contra Staphylococcus aureus, Bacillus subtilis y Pseudomonas aeruginosa; [15] y antifúngicas [20]. Por otro lado, es utilizado en el campo agrícola como pesticida, y agente de control de otros vegetales que se constituyen como flora acompañante [13].

Carbón de bambú. Este, se caracteriza por su alta porosidad y su gran capacidad de absorción, que le permiten desodorizar, desinfectar, purificar, absorber la humedad e inclusive bloquear ondas electromagnéticas. Su principal aplicación comercial, alrededor del mundo, es la producción del carbón activado de bambú [15].

Las hojas de bambú. A partir de las hojas de bambú, se pueden extraer compuestos con actividad antioxidante, comúnmente denominados AOB (antioxidantes del bambú), los cuales tienen importantes propiedades bioquímicas y metabólicas tales como la capacidad de bloquear reacciones en cadena de auto-oxidación lipídica, la captura de nitratos [17] y su extensivo uso en la preservación de alimentos cárnicos como pescados y aceites en el mercado Chino; además de su capacidad de disminuir efectivamente la producción de acrilamida (agente toxico, mutagénico y carcinogénico [20][26].

Los bambúes comestibles. Los brotes de bambú son un elemento fundamental en la comida oriental, especialmente la comida china. Además de su expresa rentabilidad en el mercado norteamericano, se destacan por sus propiedades alimenticias y gran contenido de potasio. [14][27]. 
Nubia Edith Céspedes Prieto - Juan Carda Castello

Luis Carlos Cervantes Estrada - Jonnathan Manuel Gil Noreña

\section{ANÁLISIS Y RESULTADOS}

Teniendo en cuenta el complejo escenario que plantea el posconflicto dentro del marco del Acuerdo Final para La Terminación del Conflicto y La Construcción de una Paz Estable y Duradera en Colombia y en consideraciones relacionadas con la Reforma Rural Integral y Sustitución de Cultivos Ilícitos, aquí se visualiza una propuesta novedosa y viable de sustitución voluntaria de cultivos de coca por cultivos de G. angustifolia, que involucra a las comunidades de Tumaco, Nariño afectadas por la guerra y el narcotráfico en la región del litoral Pacífico colombiano.

El incremento de cultivos ilícitos en las regiones fronterizas de Colombia, entre las que la región de Nariño repunta, es un problema que trasciende las fronteras nacionales y pone en riesgo, no sólo la implementación y el éxito de los acuerdos de Paz, sino el bienestar y el porvenir de todas las comunidades involucradas en el desarrollo del conflicto. Por esta razón de seguridad es importante que de un lado, instituciones como la Policía Nacional, hagan su participación activa en las comunidades y cuenten con mayor presencia del Gobierno Nacional, y de otro lado, las diversas organizaciones comunitarias y entidades territoriales velen por la participación de la ciudadanía en la implementación de los acuerdos, delimitación de zonas para la agricultura y reemplazo por cultivos sostenibles (o lo que es lo mismo, el Desarrollo Alternativo -DA- [9], siempre basados en la sustitución voluntaria de cultivos ilícitos, basados en la educación y el acompañamiento de la Policía Nacional. Para estas tareas el PNIS y las ZRC serán importantes estrategias de articulación, en este caso, se propone su inserción en las zonas y veredas de Tumaco destinadas al cultivo de la guadua.

Es importante realizar un acercamiento con las comunidades y establecer un censo para evaluar la fuerza laboral y la disponibilidad del terreno para el cultivo y posterior distribución y comercialización de la guadua y sus derivados potenciales. Según la experiencia de los últimos años, con respecto a la erradicación voluntaria no debemos descartar la erradicación forzada, sobre todo en aquellas zonas dominadas por bandas criminales, paramilitares y grupos disidentes guerrilleros. Para este proceso será clave la articulación de las fuerzas armadas y la Policía Nacional, que como vimos, en la primera parte de esta propuesta, estará acompañando a las comunidades a través de su policía comunitaria. Y no desconocemos esta realidad, ya que, de los registros obtenidos de erradicación forzada, Cauca (28), Nariño (25), Caquetá (24) y Antioquia (17), fueron los departamentos con mayores casos[3].

Producto de la revisión documental es posible evidenciar nuevos campos de aplicación de la $G$. angustifolia kunt más allá del nivel de infraestructura se identifican campos prometedores en el sector industrial como: la incursión en el entorno farmacéutico, de prospección médica, culinaria, de conservación ambiental y desarrollo y control agrícola, además de biorremediación por medio de productos tales como el carbón activado; aun en el campo de infraestructura, los laminados, aglomerados, aplicaciones innovadoras tales como el desarrollo de composites donde se involucran elementos como las arcillas, los polímeros y otras fibras como el kevlar en diferentes proporciones con el fin de obtener nuevas propiedades en los materiales antibalíticos, y el desarrollo de fibras textiles que le brindan a las prendas mayor confort y que para el caso de la Policía Nacional de Colombia se convierte en un potencial, porque la elaboración de los uniformes con éste tipo de fibras, favorece el servicio, debido a las distintas condiciones climáticas que debe afrontar el uniformado en las regiones del país bien sea por calor o frio, se observa en los tejidos elaborados con fibras de guadua propiedades antisépticas y de aireación que serían fácilmente aprovechables. De esta manera estas aplicaciones permiten reconocer innovaciones con potencial de desarrollo industrial que ofrecen los derivados de la guadua para el desarrollo económico de las regiones.

La guadua como una alternativa viable y sostenible a ser implementada en la región de Tumaco, Nariño, por ser el departamento que mantiene los niveles más altos de producción de cultivos ilícitos; y que requiere de una estrategia de desarrollo territorial de mayor impacto, encontrando que desde estudios, trabajo en investigación desarrollo e innovación de nuevos productos derivados de la guadua tales como: fibras textiles, brotes para proveer alimento, derivados farmacéuticos, materiales compuestos con contenido de guadua para mejorar la resistencia de los materiales, producción de carbón activado para procesos de purificación, sistemas de cultivo que aporten a la disminución de la huella de carbono. 


\section{Nubia Edith Céspedes Prieto - Juan Carda Castello \\ Luis Carlos Cervantes Estrada - Jonnathan Manuel Gil Noreña}

En Colombia se han desarrollado alternativas orientadas a la sustitución de cultivos tales como la reforestación que es considerada una oportunidad para la recuperación de los bosques que ha dejado el uso del suelo para cultivos de G. angustifolia kunt, otra perspectiva se orienta como parte del desarrollo rural basada en una economía legal, por lo que se requiere de éste tipo de estudios para los territorios que permitan visualizar no solo la producción de la materia prima sino, la oportunidad del valor agregado que se le otorga a partir de los subproductos que para el caso de estudio ofrece la guadua. Pero así como existe la esta planta son más los cultivos legales que se pueden implementar en las otras regiones deterioradas, ambiental, social y económicamente por la economía que dejan los cultivos ilícitos.

Teniendo en cuenta los acuerdos de paz de 2016 y a los puntos abarcados en la Reforma Rural Integral y Sustitución de Cultivos Ilícitos, se hace necesario el desarrollo de propuestas alternativas y viables destinadas a dar una solución a las comunidades en conflicto para que se encaren soluciones definitivas al problema de las drogas ilícitas que plantea el posconflicto.

Con el objetivo de implementar con éxito esta estrategia se traza un plan basado en cuatro ejes: 1) Participación activa de las comunidades en la erradicación voluntaria de cultivos de coca (autoridades locales, ZRC, asociaciones de productores y líderes comunitarios 2) Reforzamiento de las condiciones de seguridad en las zonas de sustitución por parte de la presencia estatal y de la policía nacional 3) Estudio del terreno para la socialización e implementación del cultivo alternativo de la guadua y 4) Monitoreo y acompañamiento constante de las fuerzas de seguridad y el Estado a las comunidades para que comercialicen sus productos y deriven del cultivo de la guadua una opción de sustento económico permanente y duradero. 4, Articulación con plan de economía naranja, de emprendedores o del sector industrial que apoye las iniciativas que se presentan.

\section{CONCLUSIÓN}

En el presente estudio genera una novedosa propuesta de desarrollo territorial para Tumaco. Nariño basada en el cultivo sostenible de Guadua angustifolia Kunth como estrategia para la sustitución voluntaria de cultivos ilícitos en una de las regiones más afectadas por esta problemática nacional en los últimos años.

El éxito para la erradicación de cultivos ilícitos se fundamente en estudios rigurosos de las condiciones de la región para la producción de materias primas de manera sostenible y el desarrollo de estudios novedosos que le den valor agregado para ser producidos y comercializados en la región con el apoyo del gobierno local, el liderazgo de la comunidad y las garantías de seguridad que les de la Policía Nacional de Colombia.

Por último, teniendo en cuenta que el mercado nacional y mundial del bambú ha mostrado una creciente demanda en los últimos años, la guadua podría configurarse en una parte importante y sostenible dentro de la economía colombiana y la erradicación de los cultivos ilícitos. Desde la Policía se hace un llamado al país y a la ciudadanía para que conozcan, apoyen y promuevan éste tipo de iniciativas regionales de desarrollo innovador destinadas a generar modelos alternativos y sostenibles de crecimiento y desarrollo de las comunidades, de cara a la construcción de un país seguro y en paz.

\section{REFERENCIAS}

[1] Presidencia de la República and FARC-EP, "ACUERDO DE PAZ EN COLOMBIA", Acuerdo final para la terminación del conflicto y la construcción de una paz estable y duradera. pp. 1-310, 2016, [Online]. Available: http://www.altocomisionadoparalapaz. gov.co/procesos-y-conversaciones/Documentos compartidos/24-11-2016NuevoAcuerdoFinal.pdf.

[2] Proyecto de Ley "Políticas públicas y paz territorial: perspectivas sobre la incidencia de la comunidad internacional a partir de los puntos 1 y 2 de los acuerdos de La Habana en el norte del Cauca 2016- 2017", $D k$, vol. 53, no. 9, pp. 1689-1699, 2017, doi: 10.1017/ CBO9781107415324.004.

[3] UNDOC, Monitoreo de territorios afectados por cultivos ilícitos 2016. 2018.

[4] R. Bonnet, J. J. Corral, and M. Barrera, "Acuerdo de paz: reforma rural, cultivos ilícitos, comunidades y costo fiscal", Bogotá DC. Investig. Econmica y Soc., pp. 1-123, 2017. 
Nubia Edith Céspedes Prieto - Juan Carda Castello

Luis Carlos Cervantes Estrada - Jonnathan Manuel Gil Noreña

[5] E. B. Elizondo, "Desarrollo de un modelo de producción de bambú guadua mediante la aplicación de técnicas silviculturales óptimas para la cuantificación del crecimiento y la capacidad de almacenamiento de carbono en plantaciones (Guadua angustifolia) en la Zona Sur de Co.", 2015.

[6] X. Londoño, F. Que, H. A. N. Contribuido, A. L. Desarrollo, D. E. L. Cultivo, and D. E. Guadua, "El bambú en Colombia", vol. 11, no. 3, pp. 143-154, 2011.

[7] César Leonardo Ardila Pinilla, "Determinación de los valores de esfuerzos admisibles del bambú", p. 93, 2013, [Online]. Available: http://www.bdigital.unal.edu.co/12616/1/ ardilapinillacesar. 2013.pdf.

[8] A.P. G. Vivianan Marcela García Valencia, Kelly Johanna Medina Suarez, Indicadores de Evaluación de Sostenibilidad Ambiental a Partir del Uso de la Guadua en Viviendas en Colombia. 2016.

[9] R. Bonnet, J. J. Corral, and M. Barrera, "Junguito Perfetti del \& Delgado Acuerdo de paz: reforma rural, cultivos ilícitos, comunidades y costo fiscal", Bogotá DC Investig. Económica y Soc., pp. 1-123, 2017.

[10] D. L. E. Y. Número, "REPUBL1CA DE COLOMBIA PRESIDENCIA DE LA REPÚBLICA", pp. 1-13, 2017.

[11] Fundación Paz y Reconciliación, "Como va la paz", Fund. Paz y Reconciliación, p. 202, 2018, [Online]. Available: https:// pares.com.co/wp-content/uploads/2018/06/ INFORME-FINAL-2018-ilovepdf-compressed.pdf.

[12] M. Borja, "Perspectivas territoriales del acuerdo de paz," Anal. Polit., vol. 30, no. 90, pp. 61-76, 2017, doi: 10.15446/anpol. v30n90.68556.

[13] P. Guznay, "Guadua Angustifolia", Palma Trop. S.A., vol. 60, no. 61, p. 4, 2004, [Online]. Available: http://www.4shared.com/ office/7cBG17R7/guadua_angustifolia_por_guzn.htm?locale=es.

[14] A. I. Mejía G et al., "Plantas del género Bambusa: Importancia y aplicaciones en la industria farmacéutica, cosmética y alimentaria", Vitae, vol. 16, no. 3, pp. 396-405, 2009.
[15] A. Mejía G et al., "Plantas del género Bambusa: importancia y aplicaciones en la industria farmacéutica", Vitae Rev. La Fac. Química y Farm., vol. 16, no. 3, pp. 396-405, 2009.

[16] D. Márquez de Hernández, Liliana; Marín Ch., "PROPAGACIÓN Y CRECIMIENTO DE Guadua amplexifolia," Bioagro, no. 1316-3361, pp. 191-198, 2011.

[17] M. Á. Ramírez-Niño and M. Á. NavarroRamirez, "Análisis de metales pesados en suelos irrigados con agua del río Guatiquía. (Heavy Metal Analysis on Soils Irrigated with Water from the Guatiquía River)", Ciencia En Desarrollo, vol. 6, no. 2, pp. 167-175, 2015, doi: 10.19053/01217488.3787.

[18] J. A. Osorio, J. M. Vélez, and H. J. Ciro, "Determinación de la relación de Poisson de la Guadua angustifolia Kunth a partir de procesamientos de imágenes y su relación con la estructura interna", Rev. Fac. Nac. Agron. Medellín, vol. 60, no. 2, pp. 4067-4076, 2007, [Online]. Available: http:// www.scielo.org.co/scielo.php?pid=S030428472007000200013\&script=sci_arttext.

[19] L. Helena, S. Villamizar, P. V. Samper, J. Manuel, and Á. Villegas, "de la Guadua en Colombia", 2012.

[20] E. S. D. Álvarez, C. G. Cabrera, and A. C. Contreras, "Estudios para el aprovechamiento potencial de hojas de guadua angustifolia kunth (poaceae), para el sector cosmético", Rev. Cuba. Farm., vol. 49, no. 3, pp. 535-542, 2015.

[21] V. Cárdenas, M. Gómez, and L. Padilla, "Degradación de celulosa y producción de etanol a partir del aserrín de guadua utilizando el sistema biológico (Aspergillus nige -Saccharomyces cerevisia)", Siquia, pp. 1-9, 2009.

[22] J. J. Murcia-Mesa et al., "Methylene blue degradation over $\mathrm{M}-\mathrm{TiO} 2$ photocatalysts $(\mathrm{M}=$ Au or Pt)", Ciencia En Desarrollo, vol. 8, no. 1, pp. 109-117, 2017, doi: 10.19053/01217488. v8.n1.2017.5352.

[23] M. B. Folgueras, F. J. Fernández, C. R. Ardila, M. Alonso, and S. Lage, "Fast pyrolysis of Guadua angustifolia-Kunth", Energy Procedia, vol. 136, pp. 60-65, 2017, doi: 10.1016/j.egypro.2017.10.283. 
Nubia Edith Céspedes Prieto - Juan Carda Castello

Luis Carlos Cervantes Estrada - Jonnathan Manuel Gil Noreña

[24] R. Izquierdo, L. Novillo, and J. Mocha, "Materiales Nobles de la Naturaleza: Caso Caña Guadua de Olon Provincia de Santa Elena Ecuador", Univ. y Soc., vol. 9, no. 2, pp. 313-318, 2017.

[25] A. Inés, M. Gallón, G. Ramírez López, H. Darío, and P. Torres, "Identificación de compuestos volátiles del vinagre de Guadua angustifolia Kunth. (guadua) Identification of volatile compounds in vinegar from Guadua angustifolia Kunth. (guadua)", Rev. Cuba. Plantas Med., vol. 16, no. 2, pp.
190-201, 2011, [Online]. Available: http:// scielo.sld.cu.

[26] L. Chan, W. Cheung, and G. Mckay, "Adsorption of acid dyes by bamboo derived activated carbon", Desalination, vol. 218, no. 1-3, pp. 304-312, Jan. 2008, doi: 10.1016/j. desal.2007.02.026.

[27] M. T. Mauricio, "Microindustria-escuela de brotes comestibles ( Bamboo Shoots ). Una estrategia para combatir la pobreza rural en la Sierra Nororiental del Estado de Puebla, México", 11th World Bamboo Congr., 2018. 
